\title{
On the rank of a random binary matrix
}

\author{
Colin Cooper* \\ Department of Computer Science \\ Kings College \\ London WC2R 2LS \\ U.K. \\ cooper.colin@gmail.com
}

\author{
Alan Frieze ${ }^{\dagger}$ Wesley Pegden ${ }^{\ddagger}$ \\ Department of Mathematical Sciences \\ Carnegie Mellon University \\ Pittsburgh PA15213 \\ U.S.A. \\ alan@random.math. cmu.edu \\ wes@math.cmu.edu
}

Submitted: Aug 14, 2018; Accepted: Sep 24, 2019; Published: Oct 11, 2019

(c) The authors. Released under the CC BY license (International 4.0).

\begin{abstract}
We study the rank of a random $n \times m$ matrix $\mathbf{A}_{n, m ; k}$ with entries from $G F(2)$, and exactly $k$ unit entries in each column, the other entries being zero. The columns are chosen independently and uniformly at random from the set of all $\left(\begin{array}{l}n \\ k\end{array}\right)$ such columns.

We obtain an asymptotically correct estimate for the rank as a function of the number of columns $m$ in terms of $c, n, k$, and where $m=c n / k$. The matrix $\mathbf{A}_{n, m ; k}$ forms the vertex-edge incidence matrix of a $k$-uniform random hypergraph $H$. The rank of $\mathbf{A}_{n, m ; k}$ can be expressed as follows. Let $\left|C_{2}\right|$ be the number of vertices of the 2-core of $H$, and $\left|E\left(C_{2}\right)\right|$ the number of edges. Let $m^{*}$ be the value of $m$ for which $\left|C_{2}\right|=\left|E\left(C_{2}\right)\right|$. Then w.h.p. for $m<m^{*}$ the rank of $\mathbf{A}_{n, m ; k}$ is asymptotic to $m$, and for $m \geqslant m^{*}$ the rank is asymptotic to $m-\left|E\left(C_{2}\right)\right|+\left|C_{2}\right|$.

In addition, assign i.i.d. $U[0,1]$ weights $X_{i}, i \in\{1,2, \ldots, m\}$ to the columns, and define the weight of a set of columns $S$ as $X(S)=\sum_{j \in S} X_{j}$. Define a basis as a set of $n-\mathbb{1}$ ( $k$ even) linearly independent columns. We obtain an asymptotically correct estimate for the minimum weight basis. This generalises the well-known result of Frieze [On the value of a random minimum spanning tree problem, Discrete Applied Mathematics, (1985)] that, for $k=2$, the expected length of a minimum weight spanning tree tends to $\zeta(3) \sim 1.202$.
\end{abstract}

Mathematics Subject Classifications: 60C05,15B52

* Research supported in part by EPSRC grant EP/M005038/1

${ }^{\dagger}$ Research supported in part by NSF Grant DMS1661063

${ }^{\ddagger}$ Research supported in part by NSF grant DMS1363136 


\section{Introduction}

Let $\Omega_{n, k}$ denote the set of vectors of length $n$, with 0,1 entries, with exactly $k 1$ 's, all other entries being zero. The addition of entries is over the field $G F_{2}$, i.e., the vector addition is over $\left(G F_{2}\right)^{n}$. Let $\mathbf{A}_{n, m ; k}$ be the random $n \times m$ matrix where the columns form a random $m$-subset of $\Omega_{n, k}$.

In a recent paper [7], we studied the binary matroid $\mathcal{M}_{n, m ; k}$ induced by the columns of $\mathbf{A}_{n, m ; k}$. It was shown that for any fixed binary matroid $M$, there were constants $k_{M}, L_{M}$ such that if $k \geqslant k_{M}$ and $m \geqslant L_{m} n$ then w.h.p. $\mathcal{M}_{n, m ; k}$ contains $M$ as a minor. The paper [7] contributes to the theory of random matroids as developed by [1], [3], [11], [13], [14]. In this paper we study a related aspect of $\mathbf{A}_{n, m ; k}$, namely its rank, and improve on results from Cooper [5]. As a consequence of the precise estimate of rank in Theorem 1 we can give an expression, (5), for the solution value of the following optimization problem.

Suppose that we assign i.i.d. $U[0,1]$ weights $X_{\mathbf{c}}$ to the vectors $\mathbf{c} \in \Omega_{n, k}$ and let the weight of a set of columns $S$ be $X(S)=\sum_{\mathbf{c} \in S} X_{\mathbf{c}}$. Define a basis as a set of $n-\mathbb{1}(k$ even) linearly independent columns. What is the expected weight $W_{n, k}$ of a minimum weight basis? When $k=2$ this amounts to estimating the expected length of a minimum weight spanning tree of $K_{n}$ which has the limiting value of $\zeta(3)$, see Frieze [8].

Our result on the rank of $\mathbf{A}_{n, m ; k}$ takes a little setting up. Let $H=H_{n, m ; k}$ denote the random $k$-uniform hypergraph with vertex set $[n]$ and $m$ random edges taken from $\left(\begin{array}{c}{[n]} \\ k\end{array}\right)$. There is a natural bijection between $\mathbf{A}_{n, m ; k}$ and $H_{n, m ; k}$ in which column $\mathbf{c}$ is replaced by the set $\left\{i: \mathbf{c}_{i}=1\right\}$. The $\rho$-core of a hypergraph $H$ (if it is non-empty) is the maximal set of vertices that induces a sub-hypergraph of minimum degree $\rho$. The 2-core $C_{2}=C_{2}(H)$ plays an important role in our first theorem.

\subsection{Matrix Rank}

Notation: We write $X_{n} \approx Y_{n}$ for sequences $X_{n}, Y_{n}, n \geqslant 0$ if $X_{n}=(1+o(1)) Y_{n}$ as $n \rightarrow \infty$. Our results are asymptotic in $n, m(n)$, as $n, m \longrightarrow \infty$, whereas $k$ is a fixed positive integer.

We will use some results on the 2-core of random hypergraphs. The size of of the 2-core has been asymptotically determined, see for example Cooper [6] or Molloy [12]; we recall the basic w.h.p. results here. In random graphs $G_{n, m}=H_{n, m ; 2}$ the 2-core grows gradually with $m$ following the emergence of the first cycle of size $O(\log n)$. For $k \geqslant 3$, the 2-core is either empty or of linear size and emerges around some threshold value $\widehat{m}_{k}$. Initially above $\widehat{m}_{k}$ the 2-core has more vertices than edges, and there is a larger value $m^{*}$, around which the number of vertices and edges becomes the same. Below $m^{*}$ the rank of the 2-core grows asymptotically as the number of edges, and above $m^{*}$ as the number of vertices.

To describe the size of the 2-core, we parameterise $m$ as $m=c n / k, c=O(1)$ and consider the equation

$$
x=\left(1-e^{-c x}\right)^{k-1} .
$$


For $k \geqslant 3$, define $\widehat{c}_{k}$ by

$$
\widehat{c}_{k}=\min \left\{c: x=\left(1-e^{-c x}\right)^{k-1} \text { has a solution } x_{c} \in(0,1]\right\} .
$$

It is known that $c<\widehat{c}_{k}$ implies that $C_{2}=\emptyset$. If $c>\widehat{c}_{k}, c=O(\log n)$, let $x_{c}$ be the largest solution to $(1)$ in $[0,1]$. Then q.s. ${ }^{1}$

$$
\begin{aligned}
& || C_{2}\left|-n\left(x_{c}^{1 /(k-1)}-c x_{c}+c x_{c}^{k /(k-1)}\right)\right| \leqslant n^{3 / 4}, \\
& || E\left(C_{2}\right)\left|-n\left(c x_{c}^{k /(k-1)} / k\right)\right| \leqslant n^{3 / 4} .
\end{aligned}
$$

We note for future reference that using (1), the term $x^{1 /(k-1)}-c x+c x^{k /(k-1)}$ in (2) can be written as $1-e^{-c x}(1+c x)$.

Let $c_{k}^{*}$ be the value of $c$ for which the 2-core has asymptotically the same number of vertices and edges. More precisely, we use (2) and (3) to define $c_{k}^{*}$ by

$$
c_{k}^{*}:=\min \left\{c \geqslant \widehat{c}_{k}: x_{c}^{1 /(k-1)}-c x_{c}+c x_{c}^{k /(k-1)}=\frac{c x_{c}^{k /(k-1)}}{k}\right\} .
$$

Define $m_{k}^{*}$ by $m_{k}^{*}=c_{k}^{*} n / k$. We will prove,

Theorem 1. If $m=O(n)$ then w.h.p.

$$
\operatorname{rank}\left(\mathbf{A}_{n, m ; k}\right) \approx \begin{cases}|E(H)| & m<m_{k}^{*} \\ |E(H)|-\left|E\left(C_{2}\right)\right|+\left|C_{2}\right| & m \geqslant m_{k}^{*}\end{cases}
$$

Note that when $k=2$ we have $c_{2}^{*}=0$ and the theorem follows from the fact that an isolated tree with $t$ edges induces a sub-matrix of rank $t$ in $\mathbf{A}_{n, m ; k}$. We therefore concentrate on the case $k \geqslant 3$.

Using (2) and (3), we can express Theorem 1 directly in terms of $c$ by

Corollary 2. Suppose that $k \geqslant 3$ and $m=c n / k$. Then, w.h.p.

$$
\operatorname{rank}\left(\mathbf{A}_{n, m ; k}\right) \approx \begin{cases}m & c<c_{k}^{*} . \\ m-m x_{c}^{k /(k-1)}+n\left(x_{c}^{1 /(k-1)}-c x_{c}+c x_{c}^{k /(k-1)}\right) & c \geqslant c_{k}^{*} .\end{cases}
$$

Around $m=n\left(\log n+d_{n}\right) / k$, where $d_{n}=o(\log n)$, the remaining vertices of degree one in $H$ disappear, and $\mathbf{A}_{n, m ; k}$ has full rank up to parity, i.e., $\operatorname{rank}\left(\mathbf{A}_{n, m ; k}\right)=n^{*}$ where

$$
n^{*}=n-\mathbb{1}(k \text { even }) .
$$

Theorem 3. Suppose that $k \geqslant 3$.

(i) Given a constant $A>0$, there exists $\gamma=\gamma(A)$ such that for $m \geqslant \gamma n \log n$,

$$
\operatorname{Pr}\left(\operatorname{rank}\left(\mathbf{A}_{n, m ; k}\right)<n^{*}\right)=o\left(n^{-A}\right) .
$$

\footnotetext{
${ }^{1} \mathrm{~A}$ sequence $\mathcal{E}_{n}$ of events occurs quite surely (q.s.) if $\operatorname{Pr}\left(\neg \mathcal{E}_{n}\right)=O\left(n^{-C}\right)$ for any constant $C>0$.
} 
(ii) If $m=n\left(\log n+d_{n}\right) / k$ then

$$
\lim _{n \rightarrow \infty} \operatorname{Pr}\left(\operatorname{rank}\left(\mathbf{A}_{n, m ; k}=n^{*}\right)\right)= \begin{cases}0 & d_{n} \rightarrow-\infty \\ e^{-e^{-d}} & d_{n} \rightarrow d \\ 1 & d_{n} \rightarrow+\infty\end{cases}
$$

We can easily modify the proof of part (ii) of Theorem 3 to give the following hitting time version. Suppose that we randomly order the columns of $\mathbf{A}_{n, M ; k}$ where $M=\left(\begin{array}{l}n \\ k\end{array}\right)$. Let $\mathbf{M}_{m}$ denote the matrix defined by the first $m$ columns in this order.

$$
m_{1}=\min \left\{m: \mathbf{M}_{m} \text { has } n^{*} \text { non-zero rows }\right\} \text { and let } m^{*}=\min \left\{m: \mathbf{M}_{m} \text { has rank } n^{*}\right\} .
$$

Theorem 4. $m_{1}=m^{*}$ w.h.p.

Some time after completion of this manuscript, we learnt from Amin Coja-Oghlan of an independent proof of Theorem 1, see [2].

\subsection{Minimum Weight Basis}

The expression (5) enables us to estimate the expected optimal value to the minimum weight basis problem defined above. Suppose that we assign i.i.d. $U[0,1]$ weights $X_{\mathbf{c}}, \mathbf{c} \in$ $\Omega_{n, k}$ to the $\left|\Omega_{n, k}\right|=\left(\begin{array}{l}n \\ k\end{array}\right)$ distinct vectors with exactly $k$ unit entries, all other entries being zeroes. The weight of a set of columns $C$ is $X(C)=\sum_{\mathbf{c} \in C} X_{\mathbf{c}}$. Let $W_{n, k}$ be the minimum weight of any basis of $n^{*}=n-\mathbb{1}(k$ even) linearly independent columns, chosen from the $\left(\begin{array}{l}n \\ k\end{array}\right)$ column vectors $\mathbf{c} \in \Omega_{n, k}$. Define the random matrix $\mathbf{A}_{n, p ; k}$ to consist of the vectors $\mathbf{c} \in \Omega_{n, k}$ with weight $X_{\mathbf{c}}$ at most $p$.

We show in Section 3 below that if $W_{n, k}$ denotes the weight of a minimum weight basis then

$$
\mathbf{E}\left(W_{n, k}\right)=\int_{p=0}^{1}\left(n^{*}-\mathbf{E}\left(\operatorname{rank}\left(\mathbf{A}_{n, p ; k}\right)\right)\right) d p .
$$

Corollary 2 and Theorem 3 can be substituted into (6) to yield an asymptotic formula for $W_{m, k}$.

Theorem 5. Let $x=x(c)$ be the largest solution of $x=\left(1-e^{-c x}\right)^{k-1}$ in $(0,1]$, then

$$
\frac{n^{k-2}}{(k-1) !} \mathbf{E}\left(W_{n, k}\right) \approx c_{k}^{*}\left(1-\frac{c_{k}^{*}}{2 k}\right)+\int_{c_{k}^{*}}^{\infty}\left(e^{-c x}\left(1+\frac{(k-1) c x}{k}\right)-\frac{c}{k}(1-x)\right) d c
$$

We note the remarkable fact that, by the result of Frieze [8], for $k=2$ and with $c_{2}^{*}=0$, the expression in $(7)$ must equal $\zeta(3)$. We have numerically estimated the first few values as a function of $k$ :

\begin{tabular}{c|c|c|c|c|c|c|c|c|c}
$k$ & 2 & 3 & 4 & 5 & 6 & 7 & 8 & 9 & 10 \\
\hline$\frac{n^{k-2}}{(k-1) !} \mathbf{E}\left(W_{n, k}\right)$ & $\zeta(3) \approx 1.202$ & 1.563 & 2.021 & 2.507 & 3.003 & 3.501 & 4.000 & 4.500 & 5.000
\end{tabular}


It appears the values are getting close to $k / 2$ as $k$ grows, and this is indeed the case.

Theorem 6. For $k \geqslant 3$, and some $\varepsilon_{k},\left|\varepsilon_{k}\right| \leqslant 5$,

$$
\lim _{n \longrightarrow \infty} \frac{n^{k-2}}{(k-1) !} \mathbf{E}\left(W_{n, k}\right)=\frac{k}{2}\left(1+\varepsilon_{k} e^{-k}\right) .
$$

\section{Matrix Rank}

We study the random matrix $\mathbf{A}_{m}$ distributed as $\mathbf{A}_{n, m ; k}$, with corresponding hypergraph $H_{m}$ distributed as $H_{n, m ; k}$. We let $c=k m / n$.

The first step of our proof is to "peel off" edges of the hypergraph $H_{m}$, and thus columns of the matrix $\mathbf{A}_{m}$, containing vertices of degree 1.

In particular, we set $H_{m}:=H_{m}$, and then, recursively, so long as $H_{i}$ contains a vertex $x_{i}$ of degree 1 , then for the edge $e_{i} \ni x_{i}$ in $H_{i}$, we set

$$
\begin{aligned}
E\left(H_{i-1}\right) & =E\left(H_{i}\right) \backslash\left\{e_{i}\right\} \\
V\left(H_{i-1}\right) & =V\left(H_{i}\right) \backslash\left\{x \in e_{i} \mid \operatorname{deg}_{H_{i}}(x)=1\right\} .
\end{aligned}
$$

In a corresponding sequence $\left\{\mathbf{A}_{i}\right\}$ beginning from $\mathbf{A}_{m}$, we obtain $\mathbf{A}_{i-1}$ from $\mathbf{A}_{i}$ by removing the column $c_{i}$ corresponding to $e_{i}$, and the (at least one) rows whose only $1 \mathrm{~s}$ were in that column. Note that for all $i<m$ for which $\mathbf{A}_{i}$ is defined, we have

$$
\operatorname{rank}\left(\mathbf{A}_{i}\right)=\operatorname{rank}\left(\mathbf{A}_{i+1}\right)-1
$$

This recursion terminates at

$$
\mathbf{C}_{2}=\mathbf{A}_{m_{2}}
$$

where $m_{2}=m-m_{1}$ is the number of edges in the the 2-core of the hypergraph $H$, and moreover, we have that $H_{m_{2}}$ is precisely the 2-core of $H$. Thus we have that

$$
\operatorname{rank}\left(\mathbf{A}_{m}\right)=m_{1}+\operatorname{rank}\left(\mathbf{C}_{2}\right)
$$

We consider the cases which control the behavior of the rank of the 2-core $\mathbf{C}_{2}=H_{m_{2}}$ of $H$. We use a theorem of Pittel and Sorkin [15] which we state here for completeness. A system of $M \times N$ equations is uniformly constrained if each variable $N$ appears at least twice. The theorem of [15] as reproduced below describes the transpose of our formulation, i.e., $A$ is an $M \times N$ matrix, and thus full row rank of $A$ corresponds to full column rank of the 2-core matrix.

Theorem ([15] Theorem 2). Let $A x=b$ be a uniformly random constrained $k$ XORSAT instance with $M$ equations and $N$ variables, with $k \geqslant 3$ and $N, M \longrightarrow \infty$ with $\lim \inf M / N>2 / k$. Then, for any $\omega(N) \longrightarrow \infty$, if $M=N-\omega(N)$ then $A x=b$ is almost surely satisfiable, with satisfiability probability $1-O\left(M^{-(k-2)}+\exp (-0.59 \omega(N))\right)$, while if $M=N+\omega(N)$ then $A x=b$ is almost surely unsatisfiable, with satisfiability probability $O\left(2^{-\omega(N)}\right)$. 
Let $N=\left|V\left(C_{2}\right)\right|$ and $M=m_{2}=\left|E\left(C_{2}\right)\right|$ be the number of rows and columns of the 2-core matrix $\mathbf{C}_{2}$. The columns associated with the 2-core $\mathbf{C}_{2}$ are distributed as uniformly random, subject to each vertex/row of the 2-core being in at least two columns.

Case 1: $c<c_{k}^{*}$.

Here $M<N$. It follows from the above Theorem of Pittel and Sorkin [15], that the rank of the columns $\mathbf{c}_{m_{1}+1}, \mathbf{c}_{m_{1}+2}, \ldots, \mathbf{c}_{m}$ is $\approx M=m_{2}=m-m_{1}$.

For this case the first claim of (5), and Theorem 1, have been verified.

Case 2: $c \geqslant c_{k}^{*}$.

Here $M>N$. To prove Theorem 1 for $c \geqslant c_{k}^{*}$ we need to verify that w.h.p.

$$
\operatorname{rank}\left(\mathbf{C}_{2}\right) \approx\left|V\left(C_{2}\right)\right|
$$

In this case we need some basic facts about hypergraphs. We say a hypergraph $H$ is linear if edges only intersect in at most one vertex. We define a $k$-uniform cactus as follows. A single edge is a cactus. An $(\ell+1)$-edge cactus $C^{\prime}$ is the structure obtained from an $\ell$-edge cactus $C$ with vertex set $V(C),|V(C)|=(k-1) \ell+1$ as follows. Choose $x \in V(C)$ and let $V\left(C^{\prime}\right)=V(C) \cup\left\{v_{1}, \ldots, v_{k-1}\right\}$ where $\left\{v_{1}, \ldots, v_{k-1}\right\}$ is disjoint from $V(C)$. The edge set $E\left(C^{\prime}\right)$ of $C^{\prime}$ is $E(C) \cup\left\{e^{\prime}\right\}$ where $e^{\prime}=\left\{x, v_{1}, \ldots, v_{k-1}\right\}$. We need the following simple lemma.

Lemma 7. A connected $k$-uniform simple hypergraph $C$ with no cycles is a cactus.

Proof. This can easily be verified by induction. We simply remove one terminal edge $e=\left\{v_{1}, v_{2}, \ldots, v_{k}\right\}$ of a longest path $P$. We can assume here that $v_{2}, \ldots, v_{k}$ are all of degree one, else $P$ can be extended. Deleting $e$ gives a new connected hypergraph $C^{\prime}$ which is a cactus by induction.

For a $k$-uniform linear hypergraph $H$ let $L(H)=(k-1)|E(H)|+1$.

Lemma 8. Let $H$ be a connected $k$-uniform linear hypergraph.

(a) $|V(H)| \leqslant L(H)$.

(b) $|V(H)|=L(H)$ if and only if $H$ does not contain any cycles.

(c) By deleting at most $L(H)-|V(H)|$ edges we can create a subgraph $H^{\prime}$ with $V\left(H^{\prime}\right)=$ $V(H)$ and no cycles.

Proof. We consider two cases:

Case 1: $H$ contains no cycles.

In this case, we consider a longest path of edges in $H$; that is consider a longest sequence $e_{1}, e_{2}, \ldots, e_{\ell}$ such that for each $1<i<e_{\ell}, e_{i}$ intersects $e_{i-1}, e_{i+1}$, and no other edges in the sequence. Since the path is longest and $H$ has no cycles, we know that $e_{\ell}$ intersects no edge in $H$ other than $e_{\ell-1}$.

In particular, we define a hypergraph $H^{\prime}$ with $E\left(H^{\prime}\right)=E(H) \backslash\left\{e_{\ell}\right\}$ and $V\left(H^{\prime}\right)=$ $V(H) \backslash\left(e_{\ell} \backslash e_{\ell-1}\right) . H^{\prime}$ has one fewer edge and $k-1$ fewer vertices than $H$, so we have $L(H)=|V(H)|$ by induction, proving the Lemma for this case. 
Case 2: $H$ contains a cycle $C$.

In this case, we consider an edge $e$ in a cycle $C$ of $H$. Removing the edge $e$ leaves a hypergraph on the same vertex set with one fewer edge and with at most $k-1$ connected components (counting isolated vertices as connected components). Applying the Lemma inductively to each component, we see that the sum of $L\left(H_{i}\right)$ over the $(k-1)$ components $H_{i}$ of $H \backslash e$ satisfies

$$
\sum_{i=1}^{k-1} L\left(H_{i}\right) \leqslant L(H)-(k-1)+(k-2) \leqslant L(H)-1,
$$

since removing $e$ decreases the sum by $k-1$, while the additive term in the definition of $L(H)$ inflates the sum by at most $(k-2)$ (as the number of components has increased by up to $k-2)$. On the other hand we of course have

$$
\sum_{i=1}^{k-1}\left|V\left(H_{i}\right)\right|=|V(H)| \text {. }
$$

We now apply parts (a) and (c) of the Lemma to each component by induction, and conclude that the Lemma does hold for $H$.

In the following lemma we prove a property of $H_{n, m ; k}$. It will be more convenient to work with $H_{n, p ; k}$ where $m=\left(\begin{array}{l}n \\ k\end{array}\right) p$. We use the fact that for any hypergraph property $\mathcal{H}$ that is monotone increasing or decreasing with respect to adding edges,

$$
\operatorname{Pr}\left(H_{n, m ; k} \in \mathcal{H}\right) \leqslant O(1) \operatorname{Pr}\left(H_{n, p ; k} \in \mathcal{H}\right) .
$$

This is well-known for graphs and is essentially a property of the binomial random variable, $E\left(H_{n, p ; k}\right)$, the number of edges of $H_{n, p ; k}$.

Similarly, if $\mathcal{A}$ is a matrix property that is monotone increasing or decreasing with respect to adding columns, then

$$
\operatorname{Pr}\left(\mathbf{A}_{n, m ; k} \in \mathcal{A}\right) \leqslant O(1) \operatorname{Pr}\left(\mathbf{A}_{n, p ; k} \in \mathcal{A}\right) .
$$

Lemma 9. Suppose that $m=O(n \log n)$.

(a) Let $\alpha<1$ be a positive constant. With probability $1-o\left(n^{-1}\right)$, for every set of vertices $S$ of size $\ell_{0}=\log ^{1 / 2} n \leqslant s \leqslant s_{0}=n^{1-\alpha}$ we have that $L(S) \leqslant s+\lfloor\theta s\rfloor$, where $\theta=\frac{1}{\log ^{1 / 4} n}$. Here $H[S]$ is the hypergraph of edges belonging completely to $S$.

(b) Then w.h.p., there are at most $n^{o(1)}$ vertices in cycles of size at most $\log ^{1 / 2} n$.

Proof. (a) We can use (12) here with $p=\frac{C \log n}{n^{k-1}}$ for some $C=O(1)$ satisfying $m=\left(\begin{array}{l}n \\ k\end{array}\right) p$. Let $s_{1}=s+\lfloor\theta s\rfloor+1$. The expected number of sets failing this property can be bounded by

$$
\sum_{s=\ell_{0}}^{s_{0}}\left(\begin{array}{l}
n \\
s
\end{array}\right) \sum_{L \geqslant s_{1}}\left(\begin{array}{c}
\left(\begin{array}{l}
s \\
k
\end{array}\right) \\
L /(k-1)
\end{array}\right)\left(\frac{C \log n}{n^{k-1}}\right)^{L /(k-1)}
$$




$$
\begin{aligned}
& \leqslant \sum_{s=\ell_{0}}^{s_{0}}\left(\frac{n e}{s}\right)^{s} \sum_{L \geqslant s_{1}}\left(\frac{C e s^{k} \log n(k-1)}{k ! L n^{k-1}}\right)^{L /(k-1)} \\
& \leqslant \sum_{s=\ell_{0}}^{s_{0}} \sum_{L \geqslant s_{1}}\left(C e^{2} \log n\right)^{L}\left(\frac{s}{n}\right)^{L-s}\left(\frac{s}{L}\right)^{L /(k-1)} \\
& \leqslant \sum_{s=\ell_{0}}^{s_{0}} \sum_{L \geqslant s_{1}}\left(\left(C e^{2} \log n\right)\left(\frac{s}{n}\right)^{1-s / L}\right)^{L}
\end{aligned}
$$

Let $u_{s, L}$ denote the summand in (14). Then we have

$$
\begin{array}{ll}
u_{L, s} \leqslant\left(\left(C e^{3} \log n\right)^{2 \alpha^{-1}}\left(\frac{s}{n}\right)^{\theta}\right)^{s} \leqslant n^{-(\alpha-o(1)) \theta s} & L \leqslant 2 \alpha^{-1} s . \\
u_{L, s} \leqslant\left(\left(C e^{3} \log n\right)\left(\frac{s}{n}\right)^{1-\alpha / 2}\right)^{L} \leqslant n^{-(1-o(1)) \alpha L / 2} & L>2 \alpha^{-1} s .
\end{array}
$$

Thus,

$$
\begin{aligned}
\sum_{s \geqslant \ell_{0}} \sum_{L \geqslant s_{1}} u_{s, L} & \leqslant \sum_{s=\ell_{0}}^{s_{0}} \sum_{L=s+\lceil\theta s\rceil}^{2 \alpha^{-1} s} n^{-(\alpha-o(1)) \theta s}+\sum_{s=\ell_{0}}^{s_{0}} \sum_{L \geqslant 2 \alpha^{-1} s} n^{-(1-o(1)) \alpha L / 2} \\
& \leqslant 2 \alpha^{-1} s_{0} \sum_{s=\ell_{0}}^{s_{0}} n^{-(\alpha-o(1)) \theta s}+\sum_{s=\ell_{0}}^{s_{0}} n^{-(1-o(1)) s / 2} \\
& =o\left(n^{-1}\right) .
\end{aligned}
$$

(b) The expected number of vertices in small cycles can be bounded by

$$
\sum_{\ell=2}^{\log ^{1 / 2} n}\left(\begin{array}{c}
n \\
(k-1) \ell
\end{array}\right)((k-1) \ell) ! p^{\ell} \leqslant \sum_{\ell=2}^{\log ^{1 / 2} n}\left(n^{k-1} p\right)^{\ell} \leqslant \sum_{\ell=2}^{\log ^{1 / 2} n}(C \log n)^{\ell}=n^{o(1)} .
$$

Part (b) now follows from the Markov inequality.

\subsection{Growth of the mantle}

We now consider the change in the rank of the sub-matrix $\mathbf{C}_{2}$ of the edge-vertex incidence matrix $\mathbf{A}_{m}$ (see (9)) corresponding to the 2-core of the column hypergraph, caused by adding a column to $\mathbf{A}_{m}$. In this section, we will assume in our calculations that no two edges share more than one vertex, and that the 2-core consists of a single connected component. This does not affect our asymptotic analysis because simple first-moment calculations show that:

1. There are only a bounded number of edges sharing more than one vertex, and 
2. Any subset of the random hypergraph of minimum degree at least 2 must be of linear size; together with (16), below, this then implies that the 2-core can only have one connected component in the present regime, since the appearance of another component at any state would increase the size of the 2-core by too much.

So suppose now that the addition of $e$ increases the size of the 2-core. Let $A$ denote the set of additional vertices and $F$ denote the set of additional edges added to $C_{2}$ by the addition of $e$, where $A \subset V(F)$. We include $e$ in $F$.

We remark first that with $c, x$ as in (1), then (2) and (3) state that q.s.

$$
|| C_{2}\left|-n\left(x_{c}^{1 /(k-1)}-c x_{c}+c x_{c}^{k /(k-1)}\right)\right| \leqslant n^{3 / 4}, \quad \text { and } \quad|| E\left(C_{2}\right)\left|-m x^{k /(k-1)}\right| \leqslant n^{3 / 4} \text {. }
$$

Therefore we can assume that adding an edge to $\mathbf{A}_{m}$ can only increase $C_{2}, E\left(C_{2}\right)$ by at most $O\left(n^{3 / 4}\right)$. We use Lemma 9 with $\alpha=3 / 4$ in our discussion of the hypergraph $F$.

Obviously the increase in rank from adding $F$ to the 2-core is bounded above by the size of the vertex-set $A$. To bound it from below, we proceed as follows:

Case 1: First consider the case where there are no cycles in $F$. We will show that the rank increases by precisely the number of new vertices.

Let $|A|=k$. We will define an ordering $a_{1}, \ldots, a_{k}$ of $A$ and a corresponding ordering $f_{1}, \ldots, f_{k}$ of a subset of $F$. To begin, we claim there must exist $v \in A$ and $v \in f \in F$, $f \neq e$, such that $f \backslash\{v\} \subseteq C_{2}$. For this consider a longest path $e_{1}, \ldots, e_{\ell}$ of edges in $F$. Since the hypergraph is simple and contains no cycles, we have that $e_{\ell} \cap\left(\bigcup_{i=1}^{\ell-1} e_{i}\right)=$ $e_{\ell} \cap e_{\ell-1}=\{v\}$ for some single vertex $v$. On the other hand, all vertices of $e_{\ell}$ must have degree 2 in $F \cup C_{2}$, and so $e_{\ell} \backslash v$ must lie entirely in $C_{2}$. We set $f_{1}=e_{\ell}, a_{1}=v$, and then we remove $f_{1}$ from $F$ and $a_{1}$ from $A$, defining $C_{2}^{1}=C_{2} \cup f_{1}$ (though it is not a two-core of any hypergraph), and apply induction to obtain the sequences $a_{1}, \ldots, a_{k}, f_{1}, \ldots, f_{k}$, and the corresponding sequence $C_{2}^{i}$ defined by $C_{2}^{0}=C_{2}$, and $C_{2}^{i+1}=C_{2}^{i} \cup f_{i+1}$.

These sequences have the property that

$$
\operatorname{rank}\left(C_{2}^{i+1}\right)=\operatorname{rank}\left(C_{2}^{i}\right)+1
$$

since the edge $f_{i}$ added to $C_{2}^{i}$ in step $i+1$ contains exactly one vertex outside of $C_{2}^{i}$. (In the matrix, we are adding a column containing a 1 in a row which previously had no 1 's).

In particular, the rank in this case increases by exactly the size of $A$.

Case 2: The total contribution to the rank of the 2-core in $m=O(n \log n)$ steps from the case where $F$ contains a cycle of length at most $\log ^{1 / 2} n$ can be bounded by $n^{3 / 4+o(1)}$. This follows from Lemma 9 (b) and (16). This is negligible, since the core has size $\Omega(n)$ in the regime we are discussing.

Case 3: Suppose that $F$ contains cycles of size at least $\log ^{1 / 2} n$ which we remove by deleting $s$ edges. When we do this we may lose up to $k s$ vertices from $A$. Let the resulting vertex set be $A^{\prime}$ and edge set be $F^{\prime}$. Up to $k s$ vertices of $A^{\prime}$ may have degree 1 . Attach these vertices to $C_{2}$ using disjoint edges to give edge set $F^{\prime \prime}$. All vertices of $A^{\prime}$ now have degree at least 2 in $F^{\prime \prime}$ and $F^{\prime \prime}$ has no cycles. According to the argument in Case 1, the increase in rank due to adding $F^{\prime \prime}$ is $\left|A^{\prime}\right| \geqslant|A|-k s$ and this is at most $k s$ larger than 
the increase in rank due to adding $F^{\prime}$. Thus the increase in rank due to adding $F \supseteq F^{\prime}$ is at least $|A|-2 k s$ and at most $|F| \leqslant|A|+s+1$. It follows from Lemma 8(c) and Lemma 9 (a) that $s=O\left(|A| / \log ^{1 / 4} n\right)$.

In summary we find that if $m=O(n \log n)$ and $m \geqslant c^{*} n / k$ then, with probability $1-o\left(n^{-1}\right)$, the rank of $\mathbf{C}_{2}$ satisfies

$$
\left(1-O\left(1 / \log ^{1 / 4} n\right)\right)\left|C_{2}\right| \leqslant \operatorname{rank}\left(\mathbf{C}_{2}\right) \leqslant\left|C_{2}\right| .
$$

The upper bound follows because the rank of $\mathbf{C}_{2}$ is at most the number of rows in $\mathbf{C}_{2}$. This proves (11). To finish the proof of Theorem 1 we require that (17) remains true if we take expectations. For this we use the error probability of $o\left(n^{-1}\right)$ in $(15)$.

\subsection{Proof of Theorem 3}

Proof of part (i):

Given a set of rows $S$ of size $s=|S|$, the number of choices of column (distinct edges) that have an odd number of non-zero entries in $S$ is

$$
T_{s, k}=\left(\begin{array}{l}
s \\
1
\end{array}\right)\left(\begin{array}{l}
n-s \\
k-1
\end{array}\right)+\left(\begin{array}{l}
s \\
3
\end{array}\right)\left(\begin{array}{l}
n-s \\
k-3
\end{array}\right)+\cdots+\left(\begin{array}{l}
s \\
k
\end{array}\right) .
$$

If $\operatorname{rank}\left(\mathbf{A}_{n, p ; k}\right)<n^{*}$ then there exists a set $S$ of rows such that (i) each column of $\mathbf{A}_{n, p ; k}$ has an even number of non-zero entries $j$ in $S$ and (ii) $|S| \leqslant n^{*}$. For a fixed $S$, denote this event by $\mathcal{B}_{S}$ and note that it is monotone decreasing. Then

$$
\operatorname{Pr}\left(\mathcal{B}_{S}\right)=(1-p)^{T_{s, k}}
$$

For $s \geqslant k$,

$$
T_{s, k} \geqslant\left(\begin{array}{l}
s \\
1
\end{array}\right)\left(\begin{array}{l}
n-s \\
k-1
\end{array}\right)+\left(\begin{array}{l}
s \\
k
\end{array}\right)=\frac{s}{(k-1) !}\left(\frac{s^{k-1}}{k}+(n-s)^{k-1}\right)(1+o(1))
$$

The bracketed term on the right hand side is minimized when $s=\alpha n$ where $\alpha=$ $k^{1 /(k-2)} /\left(1+k^{1 /(k-2)}\right)$. Let $\beta_{k}=\left(\alpha^{k-1} / k+(1-\alpha)^{k-1}\right)$ then

$$
T_{s, k} \geqslant \beta_{k} s \frac{n^{k-1}}{(k-1) !}(1+o(1)) \text {. }
$$

We can choose $p=\frac{(A+2) \log n}{\beta_{k}\left(\begin{array}{c}n-1 \\ k-1\end{array}\right)}$ and then use monotonicity of rank as a function of $p$ to claim the result for larger $p$.

$$
\begin{aligned}
\operatorname{Pr}\left(\exists S: \mathcal{B}_{S} \text { occurs }\right) & \leqslant \sum_{s=1}^{n^{*}}\left(\begin{array}{c}
n \\
s
\end{array}\right)(1-p)^{T_{s, k}} \\
& \leqslant \sum_{s=1}^{n^{*}}\left(\frac{n e}{s} \cdot \exp \left\{-p \beta_{k} \frac{n^{k-1}}{(k-1) !}(1+o(1))\right\}\right)^{s}
\end{aligned}
$$




$$
\leqslant \sum_{s=1}^{n^{*}} n^{-(A+1+o(1)) s}=O\left(\frac{1}{n^{A+1+o(1)}}\right) .
$$

We use (13) to transfer this bound to $\mathbf{A}_{n, m ; k}$.

Proof of part (ii).

Let $m=n\left(\log n+c_{n}\right) / k$. We first observe that if $Z_{s}$ denotes the number of sets of $s=O(1)$ empty rows then

$$
\begin{aligned}
& \mathbf{E}\left(Z_{s}\right)=\left(\begin{array}{c}
n \\
s
\end{array}\right) \frac{\left(\begin{array}{c}
n-s \\
k \\
m
\end{array}\right)}{\left(\begin{array}{c}
n \\
k \\
k
\end{array}\right)}=\left(\begin{array}{c}
n \\
s
\end{array}\right) \prod_{i=0}^{m-1} \frac{\left(\begin{array}{c}
n-s \\
k
\end{array}\right)-i}{\left(\begin{array}{c}
n \\
k
\end{array}\right)-i}=\left(\begin{array}{c}
n \\
s
\end{array}\right)\left(\frac{\left(\begin{array}{c}
n-s \\
k
\end{array}\right)}{\left(\begin{array}{c}
n \\
k
\end{array}\right)}\right)^{m}\left(1+O\left(\frac{m^{2}}{n^{k}}\right)\right) \\
& \approx \frac{n^{s}}{s !} \cdot \prod_{i=0}^{k-1}\left(1-\frac{s}{n-i}\right)^{m}=\frac{n^{s}}{s !} \cdot \prod_{i=0}^{k-1} \exp \left\{-\frac{m s}{n}+O\left(\frac{m}{n^{2}}\right)\right\} \approx \frac{n^{s}}{s !} e^{-s k m / n} \approx \frac{e^{-c s}}{s !} .
\end{aligned}
$$

Thus if $c_{n} \longrightarrow \infty, \mathbf{E}\left(Z_{1}\right) \longrightarrow 0$, and if $c_{n} \longrightarrow-\infty, \mathbf{E}\left(Z_{1}\right) \longrightarrow \infty$. Straightforward arguments complete Theorem 3(ii) for these cases.

Assume next that $c_{n} \rightarrow c$. The method of moments applied to (20) implies that $Z_{1}$ is asymptotically Poisson with mean $e^{-c}$ and so

$$
\operatorname{Pr}\left(Z_{1}=0\right) \approx e^{-e^{-c}}
$$

The final step is to prove (w.h.p) that when $p=\left(\log n+c_{n}\right) /\left(\begin{array}{l}n-1 \\ k-1\end{array}\right), c_{n} \longrightarrow c$ constant, the only obstruction to $\operatorname{rank}\left(\mathbf{A}_{n, p ; k}\right)=n^{*}$ is the existence of empty rows $\left(Z_{1}>0\right)$. As in part (i) above, going back to (19) with $p=\left(\log n+c_{n}\right) /\left(\begin{array}{l}n-1 \\ k-1\end{array}\right)$ we see that we only need to consider $2 \leqslant s \leqslant e^{2+\beta_{k}\left|c_{n}\right|} n^{1-\beta_{k}}$. For these values of $s, T_{s, k}$ is bounded below by $s\left(\begin{array}{l}n-s \\ k-1\end{array}\right) \approx s\left(\begin{array}{l}n-1 \\ k-1\end{array}\right)$. Similar to the derivation of (19), we see we can bound the probability of the event $\left\{\exists S: \mathcal{B}_{S}\right\}$ from above by

$$
\operatorname{Pr}\left(\mathcal{B}_{S}\right) \leqslant\left(\frac{3 n}{s} \cdot \exp \left\{-p\left(\begin{array}{c}
n-1 \\
k-1
\end{array}\right)\right\}\right)^{s}=\left(\frac{O\left(e^{-c}\right)}{s}\right)^{s} .
$$

Thus, for $|c|$ constant, with $s_{1}=e^{2+\beta_{k}\left|c_{n}\right|} n^{1-\beta_{k}}$

$$
\operatorname{Pr}\left(\exists S, \log \log n \leqslant|S| \leqslant s_{1}: \mathcal{B}_{S} \text { occurs }\right) \leqslant \sum_{s=\log \log n}^{s_{1}}\left(\frac{O\left(e^{-c}\right)}{s}\right)^{s}=o(1) .
$$

Finally we consider $2 \leqslant s \leqslant L=\log \log n$. Given a set $S$, the number of choices of column that have an odd number of non-zero entries in $S$ (Type A columns) is given by $T_{s, k}$ above. The number of choices of columns that have an even number of non-zero entries in $S$ (Type B columns) is

$$
R_{s, k}=\left(\begin{array}{l}
s \\
2
\end{array}\right)\left(\begin{array}{l}
n-s \\
k-2
\end{array}\right)+\cdots+\left(\begin{array}{c}
s \\
k-1
\end{array}\right)(n-s) .
$$


For $s \leqslant L, R_{s, k} \leqslant s^{2} n^{k-2}$. The expected number $\mu_{s}$ of sets $S$ with no Type A columns and at least one Type B column is

$$
\mu_{s}=\left(\begin{array}{c}
n \\
s
\end{array}\right)\left(1-(1-p)^{R_{s, k}}\right)(1-p)^{T_{s, k}} \leqslant \frac{n^{s}}{s !}\left(p R_{s, k}\right) e^{-p s\left(\begin{array}{c}
n-1 \\
k-1
\end{array}\right)(1+o(1))}=O\left(\frac{\log n}{n}\right) e^{-c s} .
$$

Thus, for constant $c$,

$$
\sum_{s=2}^{L} \mu_{s}=o(1) .
$$

Thus w.h.p. there is no set of $2 \leqslant s \leqslant \log \log n$ rows where the dependency does not come from the rows all being zero.

\subsection{Proof of Theorem 4}

Because $c$ in (21) is arbitrary and having a zero row is a monotone decreasing event, we can see that if $m_{0}=n(\log n-\log \log n) / k$ then $Z_{1}=Z_{1}\left(m_{0}\right)>0$ w.h.p. The reader can easily check that equations (22) and (23) continue to hold. It follows that w.h.p. the rank of $\mathbf{M}_{m_{0}}$ is $n^{*}-Z_{1}$. It then follows that $m_{1}=m^{*}$ if we never add a column that reduces the number of non-zero rows by more than one. Now (21) implies that the expected number of zero rows in $\mathbf{M}_{m_{0}}$ is $O(\log n)$ and so $Z_{1} \leqslant \log ^{2} n$ w.h.p. So given this, the probability we add add a column that reduces the number of non-zero rows by more than one in the next $O(n \log n)$ column additions, is $O\left(n \log n \times\left(\left(\log ^{2} n\right) / n\right)^{2}=o(1)\right.$.

\section{Minimum Weight Basis}

The first task here is to prove (6). Let $B_{n, k}$ denote a minimum weight basis and let $W_{n, k}$ denote its weight. For a given a real number $X$ we can write

$$
X=\int_{p=0}^{X} d p=\int_{p=0}^{1} 1_{p \leqslant X} d p .
$$

Thus

$$
\begin{aligned}
W_{n, k} & =\sum_{\mathbf{c} \in B_{n, k}} X_{\mathbf{c}} \\
& =\sum_{\mathbf{c} \in B_{n, k}} \int_{p=0}^{1} 1_{p \leqslant X_{\mathbf{c}}} d p \\
& =\int_{p=0}^{1} \sum_{\mathbf{c} \in B_{n, k}} 1_{p \leqslant X_{\mathbf{c}}} d p
\end{aligned}
$$




$$
\begin{aligned}
& =\int_{p=0}^{1}\left|\left\{\mathbf{c} \in B_{n, k}: p \leqslant X_{\mathbf{c}}\right\}\right| d p \\
& =\int_{p=0}^{1}\left(n^{*}-\operatorname{rank}\left(\mathbf{A}_{p}\right)\right) d p .
\end{aligned}
$$

Here $\mathbf{A}_{p}$ is any matrix made up of those columns $\mathbf{c} \in \Omega_{n, k}$ with $X_{\mathbf{c}} \leqslant p$. And let $A_{p}$ denote the corresponding hypergraph.

Explanation for (25): Finding a minimum cost basis $B$ can be achieved via a greedy algorithm. We first order the columns of $\Omega_{n, k}$ as $\mathbf{c}_{1}, \mathbf{c}_{2}, \ldots, \mathbf{c}_{N}, N=\left(\begin{array}{l}n \\ k\end{array}\right)$ in increasing order of weight $X_{\mathbf{c}}$. Treating $B$ as a set of columns, we initialise $B=\emptyset$, and for $i=1,2, \ldots, N$ add $\mathbf{c}_{i}$ to $B$ if it is linearly independent of the columns of $B$ selected so far. This means that for any $0 \leqslant p \leqslant 1$, the number of columns in $B$ with $X_{\mathbf{c}}>p$ must be equal to the co-rank of the set of columns selected before them i.e $B_{p}=\left\{\mathbf{c} \in B: X_{\mathbf{c}} \leqslant p\right\}$. We claim that $B_{p}$ is a maximal linear independent subset of the columns of $\mathbf{A}_{p}$. If it were not maximal, then another column of $\mathbf{A}_{p}$ would have been added to $B_{p}$ by the greedy algorithm.

We obtain $\mathbf{E} W_{n, k}$ in (6) by taking the expectation of (25), using Fubini's theorem to take the expectation inside the integral.

We first argue that

$$
\mathbf{E}\left(W_{n, k}\right)=\Omega\left(n^{-(k-2)}\right) .
$$

Let $\mathbf{c}=\left(c_{1}, \ldots, c_{n}\right)$, where $c_{i} \in\{0,1\}$ denotes the $i$-th row coordinate of $\mathbf{c}$. We can bound $W_{n, k}$ from below by $\sum_{i=1}^{n} \min \left\{X_{\mathbf{c}}: c_{i}=1\right\} / k$. Let $N=\left(\begin{array}{l}n \\ k\end{array}\right)$. The number of ones in a fixed row of $\mathbf{A}_{n, N ; k}$ is $L=N k / n$. The expected minimum of $L$ independent uniform $[0,1]$ random variables is $1 /(L+1)$. Hence

$$
\mathbf{E}\left(W_{n, k}\right) \geqslant \frac{1}{k} \frac{n^{2}}{k\left(\begin{array}{l}
n \\
k
\end{array}\right)+n}
$$

and (26) follows.

We next observe that for $c$ large we have

$$
1-2 k e^{-c} \leqslant x \leqslant 1 .
$$

Indeed, putting $x=1-y$ in $(1)$ gives $(1-y)^{1 /(k-1)}=1-e^{-c(1-y)}$. We see that if $f(y)=(1-y)^{1 /(k-1)}-\left(1-e^{-c(1-y)}\right)$ then $f(0)>0$ and $f\left(2 k e^{-c}\right)<0$ for large $c$.

Thus for $c$ large we have

$$
\frac{c}{k}-\frac{c x^{k /(k-1)}}{k}+\left(1-e^{-c x}(1+c x)\right) \geqslant 1-e^{-c x}(1+c x) \geqslant 1-e^{-99 c / 100} .
$$

Fix some small $\varepsilon>0$ and let

$$
c_{\varepsilon}=2 \log 1 / \varepsilon .
$$

It follows from Theorem 3(i) with $A=k, p=k m /\left(n\left(\begin{array}{l}n-1 \\ k-1\end{array}\right)\right)$. and (6) that

$$
\mathbf{E}\left(W_{n, k}\right) \approx \int_{p=0}^{k ! \gamma n^{1-k} \log n}\left(n^{*}-\mathbf{E}\left(\operatorname{rank}\left(\mathbf{A}_{p}\right)\right)\right) d p
$$




$$
\begin{aligned}
& =\frac{(k-1) !}{n^{k-1}} \int_{c=0}^{k \gamma \log n}\left(n^{*}-\mathbf{E}\left(\operatorname{rank}\left(\mathbf{A}_{c(k-1) ! / n^{k-1}}\right)\right)\right) d c \\
& =\left(I_{1}+I_{2}+I_{3}\right) \frac{(k-1) !}{n^{k-1}}
\end{aligned}
$$

where $I_{1}=\int_{c=0}^{c_{k}^{*}} \cdots d c$ and $I_{2}=\int_{c_{k}^{*}}^{c_{\varepsilon}} \cdots d c$ and $I_{3}=\int_{c_{\varepsilon}}^{k \gamma \log n} \cdots d c$.

Since $H_{c / n^{k-1}}$ q.s. has $m \approx c n / k$ edges, it follows from Theorem 1 that

$$
I_{1} \approx \int_{c=0}^{c_{k}^{*}}\left(n^{*}-\frac{c n}{k}\right) d c \approx c_{k}^{*} n\left(1-\frac{c_{k}^{*}}{2 k}\right)
$$

On the other hand, using the expression for rank from Corollary 2, with $x^{1 /(k-1)}=$ $\left(1-e^{-c x}\right)$ substituted from $(1)$.

$$
\begin{aligned}
I_{2} & \approx n \int_{c_{k}^{*}}^{c_{\varepsilon}}\left(1-\left(\frac{c}{k}-\frac{c x^{k /(k-1)}}{k}+\left(1-e^{-c x}(1+c x)\right)\right)\right) d c \\
& =n \int_{c_{k}^{*}}^{\infty}\left(e^{-c x}(1+c x(k-1) / k)-\frac{c}{k}(1-x)\right) d c+A_{\varepsilon} .
\end{aligned}
$$

Using (28) gives

$$
\left|A_{\varepsilon}\right|=n \int_{c_{\varepsilon}}^{\infty}\left(e^{-c x}(1+c x(k-1) / k)-\frac{c}{k}(1-x)\right) d c \leqslant n \int_{c_{\varepsilon}}^{\infty} e^{-99 c / 100} d c \leqslant 2 \varepsilon n .
$$

Theorem 1 as stated holds for $m=O(n)$, and thus cannot be used directly to estimate rank when $m / n \longrightarrow \infty$. For $I_{3}$ we recall that $\mathbf{C}_{2}=\mathbf{C}_{2}(c)$ denotes the sub-matrix of $\mathbf{A}_{c(k-1) ! / n^{k-1}}$ induced by the edges of the 2-core. We then write

$$
I_{3} \leqslant \int_{c_{\varepsilon}}^{k \gamma \log n}\left(n^{*}-\mathbf{E}\left(\operatorname{rank}\left(\mathbf{C}_{2}(c)\right)\right)\right) d c .
$$

We first check the size of $\left|C_{2}\right|$ for $c=c_{\varepsilon}$. It follows from (1) and (28) that for $c$ large,

$$
x^{1 /(k-1)}-c x+c x^{k /(k-1)}=1-e^{-c x}(1+c x) \geqslant 1-e^{-99 c / 100} .
$$

So, for large enough $c=O(1)$, from (2) we have that w.h.p.

$$
\left|C_{2}\right| \geqslant(1-o(1)) n\left(1-e^{-99 c / 100}\right) .
$$

Let $m_{\varepsilon}=c_{\varepsilon} n / k$. If we add an edge $e$ with one vertex not in $C_{2}$ and the remaining vertices in $C_{2}$ then the rank of $\mathbf{C}_{2}$ goes up by one. Denote this event by $\mathcal{A}_{e}$. Let $\mathbf{C}^{*}=\mathbf{C}^{*}(t)$ denote the following submatrix of $\mathbf{C}_{2}$ at the time the number of columns is $m_{\varepsilon}+t$. We let $\mathbf{C}^{*}(0)=\mathbf{C}_{2}\left(c_{\varepsilon}\right)$ and we add the column corresponding to $e$ to $\mathbf{C}^{*}$ only if $\mathcal{A}_{e}$ occurs. Let $X_{t}$ denote the rank of $\mathbf{C}^{*}(t)$, and let $Y_{t}=n^{*}-X_{t}$. Note that $X_{t}$ is equal to $\operatorname{rank}\left(\mathbf{C}_{2}\left(c_{\varepsilon}\right)\right)$ plus the number of columns in $\mathbf{C}^{*}(t)$ that are not in $\mathbf{C}_{2}\left(c_{\varepsilon}\right)$, and that $X_{t} \leqslant \operatorname{rank}\left(\mathbf{A}_{m_{\varepsilon}+t}\right)$. 
Note also that $\left|\operatorname{rank}\left(\mathbf{A}_{m_{\varepsilon}+t}\right)-\operatorname{rank}\left(\mathbf{A}_{n, p_{t}, k}\right)\right| \leqslant n^{2 / 3}$ where $p_{t}=\left(m_{\varepsilon}+t\right) /\left(\begin{array}{l}n \\ k\end{array}\right)$. Using (29) we have that $Y_{0} \leqslant(1+o(1)) n e^{-99 c_{\varepsilon} / 100} \leqslant 2 \varepsilon n$. Now,

$$
\operatorname{Pr}\left(\mathcal{A}_{e}\right)=\frac{Y_{t}\left(\begin{array}{c}
n-Y_{t} \\
k-1
\end{array}\right)}{\left(\begin{array}{l}
n \\
k
\end{array}\right)} \geqslant \frac{k Y_{t}}{2 n}
$$

and so

$$
\mathbf{E}\left(Y_{t+1} \mid Y_{t}\right) \leqslant Y_{t}-\frac{k Y_{t}}{2 n} .
$$

Let $h=n^{1 / 2}$ and $u_{r}=Y_{r h}$. Assume that $n^{9 / 10} \leqslant Y_{t} \leqslant Y_{0}$. It follows from (36) and Hoeffding's Theorem [9] that q.s.

$$
u_{r+1} \leqslant u_{r}-\frac{k h}{3 n} u_{r}=\left(1-\frac{k h}{3 n}\right) u_{r}
$$

and so q.s.

$$
u_{r} \leqslant\left(1-\frac{k h}{3 n}\right)^{r} u_{0}
$$

Going back to (35) we can see that

$$
I_{3} \leqslant O\left(n^{9 / 10}\right)+\frac{h u_{0}}{n} \sum_{r=0}^{\infty}\left(1-\frac{k h}{3 n}\right)^{r}=O\left(n^{9 / 10}\right)+\frac{3 u_{0}}{k} .
$$

Here the final $O\left(n^{9 / 10}\right)$ term accounts for only using (37) for $Y_{t} \geqslant n^{9 / 10}$ and for the errors of size $O\left(n^{2 / 3}\right)$ introduced in the $m$ model versus the $p$ model of our matrix, see (12), (13).

It follows from (31), (32), (34) and (39) that $I_{1}+I_{2}+I_{3}$ are within $O(\varepsilon n)$ of what is claimed in the theorem. Since $\varepsilon$ is arbitrary, we can by increasing $c_{\varepsilon}$, make the value of $\varepsilon$ in (29) arbitrarily small and Theorem 5 follows.

\subsection{Bounds for finite $k$}

We begin by estimating $c_{k}^{*}$. Let $x$ be as in (1), then going back to the definition (4), we can determine the value of $c_{k}^{*}=c(x)$ from

$$
c\left(\frac{k-1}{k}\right) x^{\frac{k}{k-1}}-c x+x^{\frac{1}{k-1}}=0 .
$$

Solve for $c$, and put $y=x^{1 /(k-1)}$ to give

$$
c=\frac{1}{y^{k-2}-((k-1) / k) y^{k-1}} \text {. }
$$

Substituting for $c$ via (1) gives

$$
y=1-\exp \left\{-\frac{k y}{k-(k-1) y}\right\} .
$$


If $x \in(0,1)$ then $y \in(0,1)$, and $y \geqslant x$. We look for solutions of the form $y=1-z$. Making this substitution (42) becomes $z=q(z)$ where

$$
q(z)=\exp \left\{-\frac{k(1-z)}{1+(k-1) z}\right\}
$$

Let

$$
z=z(\delta)=\frac{\delta}{k-(k-1) \delta},
$$

then (stretching notation somewhat) $q(\delta)=e^{-k(1-\delta)}$. Consider $f(\delta)=z(\delta)-q(\delta)$, then

$$
f(\delta) \geqslant \frac{\delta}{k}\left(1+\frac{k-1}{k} \delta\right)-e^{-k} e^{k \delta}
$$

Substitute $\delta=\theta k e^{-k}$ to give

$$
f(\theta) \geqslant e^{-k}\left(\theta\left(1+\theta(k-1) e^{-k}\right)-e^{\theta k^{2} e^{-k}}\right) .
$$

The function $k^{2} e^{-k}$ in the exponent of the last term is monotone deceasing for $k \geqslant 2$. Let $\theta=3 / 2$, then for $k \geqslant 4$, it can be checked that $f(\theta, k)>0$. Now $f(0)<0$ and so there is a solution to $f(\delta)=0$ in the interval $\left(0, \theta k e^{-k}\right)$.

Substitute $y=1-z$ into (41) to obtain

$$
\frac{c}{k}=\frac{1}{(1-z)^{k-2}(1+(k-1) z)}
$$

Lemma 10. (i) Let $\theta=3 / 2$, then for $k \geqslant 4$,

$$
k\left(1-\theta e^{-k}\right) \leqslant c_{k}^{*} \leqslant k .
$$

(ii) For $k=3, c_{3}^{*}=2.753813 \ldots$

(iii) If $k \geqslant 4$ and $c \geqslant c_{k}^{*}$ then the solution $x$ to (1) satisfies $x \geqslant 1-3 k e^{-c} / 2$.

Proof. (i) For the upper bound we note that for $k \geqslant 3$ the denominator of $c$ in (44) is monotone increasing for $z \leqslant 1 /(k-1)^{2}$ from a value of one when $z=0$. For the lower bound, as $1 /(1-z)^{k-2}>1+(k-2) z$, it follows from (44), the definition of $z$ in (43), and $\delta<\theta k e^{-k}$ that

$$
\frac{c}{k}>\frac{1+(k-2) z}{1+(k-1) z}=1-\frac{\delta}{k}>1-\theta e^{-k} .
$$

(ii) Set $y=\sqrt{x}$ and invert (1) to obtain

$$
c=\frac{1}{y^{2}} \log \frac{1}{1-y} .
$$


Inserting this into (41) gives

$$
y+\left(\frac{2}{3} y-1\right) \log \frac{1}{1-y}=0 .
$$

This equation was solved numerically to give the following results for $y, x, c_{3}^{*}$

$$
y=0.8834191, \quad x=0.9399038, \quad c_{3}^{*}=2.753813 .
$$

(iii) Let $x=1-\varepsilon$. We first verify that $\varepsilon \leqslant 1 / c$. Putting $f(\varepsilon)=1-\varepsilon-\left(1-e^{-c+c \varepsilon}\right)^{k-1}$ we see that $f(0)>0$ and $f(1 / c)<0$ for $c \geqslant c_{k}^{*}$ as given in (i). If ay $<1$, then $1-(1-y)^{a}<a y$. As $(k-1) e^{-c+c \varepsilon}<1$ for any $\varepsilon<1-(\log (k-1)) / c$,

$$
f\left(c^{-1}\right)=1-c^{-1}-\left(1-e^{-c+1}\right)^{k-1} \leqslant 1-c^{-1}-1+(k-1) e^{-c+1} .
$$

Now $c(k-1) e^{-c+1}$ is decreasing as a function of $c$. And for $k \geqslant 4, k(k-1) e^{-c+1}$ and $e^{(3 k / 2) e^{-k}}$ are decreasing as functions in of $k$. Therefore, for $c$ satisfying (45),

$$
c(k-1) e^{-c+1}<k(k-1) e^{-(k-1)} e^{(3 k / 2) e^{-k}}<1 .
$$

Let $x=1-\varepsilon$, and $\delta=e^{-c+c \varepsilon}$. Rewrite (1) as

$$
-\log (1-\varepsilon)=\varepsilon+\frac{\varepsilon^{2}}{2}+\cdots=(k-1)\left(\delta+\frac{\delta^{2}}{2}+\cdots\right) .
$$

It must hold that $\varepsilon \leqslant(k-1) \delta$ otherwise the left hand side is greater than the right hand side. Thus, as $\varepsilon<1 / c$,

$$
\varepsilon \leqslant(k-1) e^{-c+c \varepsilon} \leqslant(k-1) e^{-c+1} .
$$

A repeated application of this bound, (45) and direct calculation gives $\varepsilon \leqslant(k-1) \exp \left\{-c+(k-1) c e^{-c+1}\right\} \leqslant(k-1) \exp \left\{-c+(k-1) k e^{1-\left(1-\theta e^{-k}\right) k}\right\} \leqslant 3 k e^{-c} / 2$.

Going back to (31) and using Lemma 10(i), we see that for $k \geqslant 4$,

$$
\frac{k n}{2}\left(1-\frac{9}{4} e^{-2 k}\right) \leqslant I_{1} \leqslant \frac{k n}{2} .
$$

We evaluate $I_{2}$ from (32)-(33) in two parts. Firstly, using Lemma 10(iii) for $c \geqslant c_{k}^{*}$,

$$
-\frac{3}{2} c e^{-c} \leqslant-\frac{c}{k}(1-x) \leqslant 0 .
$$

Note also that $1-3 k e^{-c} / 2 \geqslant 1-1 / 2 k$ for $k \geqslant 4$ and $c \geqslant c_{k}^{*}$. Thus

$$
e^{-c}\left(1+c \frac{(k-1)(2 k-1)}{2 k^{2}}\right) \leqslant e^{-c x}\left(1+c x \frac{k-1}{k}\right) \leqslant e^{1 / 2} e^{-c}\left(1+\frac{c(k-1)}{k}\right) .
$$


For the LHS we replace $e^{-c x}$ by $e^{-c}$ (since $x \leqslant 1$ ) and $x$ by $1-1 / 2 k$. For the RHS we replace $c x(k-1)$ by $c(k-1)$, and $e^{-c x}=e^{-c+c \varepsilon}$. Using Lemma 10(i) and (iii), as $c^{*}>1$, it follows that

$$
e^{c \varepsilon} \leqslant e^{(3 k / 2) c e^{-c}} \leqslant e^{(3 k / 2) c^{*} e^{-c^{*}}} \leqslant e^{1 / 2}
$$

Adding the contributions from (49) and (50) we find that

$$
n \int_{c^{*}}^{\infty} e^{-c}\left(1-c \frac{k^{2}+3 k-1}{2 k^{2}}\right) d c \leqslant I_{2} \leqslant n e^{1 / 2} \int_{c^{*}}^{\infty} e^{-c}\left(1+\frac{c(k-1)}{k}\right) d c .
$$

Thus, with the indefinite integral $\int e^{-c}(1+A c)=-e^{-c}(1+A+A c)$, we get

$$
n e^{-c_{k}^{*}}\left(\frac{k^{2}-3 k+1}{2 k^{2}}-c_{k}^{*} \frac{k^{2}+3 k-1}{2 k^{2}}\right) \leqslant I_{2} \leqslant n e^{1 / 2} e^{-c_{k}^{*}}\left(\frac{2 k-1}{k}+c_{k}^{*} \frac{k-1}{k}\right),
$$

or more simply

$$
-n \frac{k}{2} e^{-c_{k}^{*}}\left(1+\frac{3}{k}\right) \leqslant I_{2} \leqslant n \frac{k}{2} e^{-c_{k}^{*}} 2 e^{1 / 2}\left(1+\frac{1}{k}\right) .
$$

Noting that $e^{-c_{k}^{*}} \leqslant 6 e^{-k} / 5$ for $k \geqslant 4$, we have

$$
n \frac{k}{2}\left(1-\frac{9}{4} e^{-2 k}-\frac{21}{10} e^{-k}\right) \leqslant I_{1}+I_{2} \leqslant n \frac{k}{2}\left(1+3 e^{1 / 2} e^{-k}\right) .
$$

Thus, for some $\varepsilon_{k},\left|\varepsilon_{k}\right| \leqslant 5$,

$$
I_{1}+I_{2}=n \frac{k}{2}\left(1+\varepsilon_{k} e^{-k}\right)
$$

\section{Open questions}

Q1 The formula for the cost of a minimum weight basis when $k \geqslant 3$ given by Theorem 5 is asymptotically accurate, but lacks the elegance of the case where $k=2$. Can the expression be simplified for say, $k=3$ ?

Q2 The $\zeta(3)$ result of [8] was generalised quite substantially to consider minimum weight spanning trees of $d$-regular graphs, when $d$ is large, see [4]. In the context of $\mathbf{A}_{n, m ; k}$, this suggests that we consider the case where each row has exactly $d$ ones. Here we can study the rank as well as $W_{n, k}$.

\section{References}

[1] J. Altschuler and E. Yang, Inclusion of Forbidden Minors in Random Representable Matroids, Discrete Mathematics 340 (2017) 1553-1563. 
[2] P. Ayre, A. Coja-Oghlan, P. Gao and N Müller, The satisfiabilty threshold for random linear equations, arXiv:1710.07497.

[3] N. Bansal, R.A. Pendavingh and J.G. van der Pol, On the number of matroids, Combinatorica 35 (215) 253-277.

[4] A. Beveridge, A. M. Frieze and C. J. H. McDiarmid, Minimum length spanning trees in regular graphs, Combinatorica 18 (1998) 311-333.

[5] C. Cooper, On the rank of random matrices, Random Structures and Algorithms 16 (2000) 209-232.

[6] C. Cooper, The cores of random hypergraphs with a given degree sequence, Random Structures and Algorithms 25 (2004) 353-375.

[7] C. Cooper, A.M. Frieze and W. Pegden, Minors of a random binary matroid, arXiv:1612.02084v5.

[8] A.M. Frieze, On the value of a random minimum spanning tree problem, Discrete Applied Mathematics 10 (1985) 47-56.

[9] W. Hoeffding, Probability inequalities for sums of bounded random variables, Journal of the American Statistical Association 58 (1963) 13-30.

[10] S. Janson, The minimal spanning tree in a complete graph and a functional limit theorem for trees in a random graph, Random Structures and Algorithms 7 (1995) 337-355.

[11] W. Kordecki and A. Lyczkowska-Hanćkowiak, Exact Expectation and Variance of Minimal Basis of Random Matroids, Discussiones Mathematicae Graph Theory 33 (2013) 277-288.

[12] M. Molloy, Cores in random hypergraphs and random formulas, Random Structures and Algorithms 27 (2005) 124-135.

[13] J. Oxley and D. Kelly, On random representable matroids, Studies in Applied Mathematics 71 (1984) 181-205.

[14] J. Oxley, L. Lowrance, C. Semple and D. Welsh, On properties of almost all matroids, Advances in Applied Mathematics 50 (2013) 115-124.

[15] B. Pittel and G. Sorkin, The Satisfiability Threshold for $k$-XORSAT, Combinatorics, Probability and Computing 25 (2016) 238-268. 